\title{
Nivolumab in the treatment of malignant melanoma: review of the literature
}

REVIEW

\author{
This article was published in the following Dove Press journal: \\ OncoTargets and Therapy \\ 6 August 2015 \\ Number of times this article has been viewed
}

\author{
Emi Mashima \\ Akiha Inoue \\ Yumiko Sakuragi \\ Takashi Yamaguchi \\ Natsuko Sasaki \\ Yoko Hara \\ Daisuke Omoto \\ Shun Ohmori \\ Sanehito Haruyama \\ Yu Sawada \\ Manabu Yoshioka \\ Daisuke Nishio \\ Motonobu Nakamura \\ Department of Dermatology, \\ University of Occupational \\ and Environmental Health, \\ Kitakyushu, Japan
}

Correspondence: Motonobu Nakamura Department of Dermatology, University of Occupational and Environmental Health, I-I Iseigaoka, Yahatanishi-ku, Kitakyushu 807-8555, Japan

Tel +8I 936917445

Fax +8I 93 69I 0907

Email motonaka@med.uoeh-u.ac.jp

\begin{abstract}
Nivolumab was developed as a monoclonal antibody against programmed death receptor-1, an immune checkpoint inhibitor which negatively regulates T-cell proliferation and activation. Intravenous administration of nivolumab was approved for the treatment of unresectable malignant melanoma in 2014 in Japan. When advanced melanoma patients were treated with nivolumab, median overall survival became longer. Overall survival rate was significantly better in nivolumab-treated melanoma patients than dacarbazine-treated melanoma patients. Nivolumab had an acceptable long-term tolerability profile, with $22 \%$ of patients experiencing grade 3 or 4 adverse events related to the drug. Therefore, nivolumab can become an alternative therapy for advanced malignant melanoma.
\end{abstract}

Keywords: monoclonal antibody, PD-1, PD-L1

\section{Introduction}

Malignant melanoma is a melanocyte neoplasm that usually takes place in the skin and occasionally harbors mutations in genes including $B R A F .^{1,2}$ Each year in the world, the number of estimated new cases of malignant melanoma is 132,000 and approximately 48,000 patients die from malignant melanoma. When malignant melanoma is diagnosed at the early stage (stage 0/I), 5-year survival rate is more than $90 \%$ after surgical excision. However, when malignant melanoma is overlooked, it tends to invade deeply and metastasize to lymph nodes and other organs. Median overall survival rate for melanoma patients with metastasis is less than 1 year.

For advanced melanoma, systemic therapy is usually needed; however, there were only limited options just a few years ago. There are a lot of patients who are resistant to conventional chemotherapies with an alkylating agent, dacarbazine, and interferon (IFN)- $\alpha$.

There have been a lot of studies on development of novel therapies for unresectable malignant melanoma targeting immune checkpoint inhibitors. ${ }^{3,4}$ One of the recent advancements came from the new development of monoclonal antibody against an immune checkpoint inhibitor, programmed death receptor-1 (PD-1). ${ }^{5,6}$

Nivolumab was developed as a fully human IgG4 monoclonal antibody against PD-1, an immune checkpoint inhibitor that negatively controls T-cell proliferation and functions. Intravenous administration of nivolumab was approved for the treatment of unresectable malignant melanoma in 2014 in Japan. This review focuses on the functional and molecular characteristics of PD-1 and the clinical efficacy and tolerability of its antibody, nivolumab.

\section{PD-I and its ligands: PD-LI and PD-L2}

The PD-1 gene was first cloned and its nucleotide sequence was reported by Professor Honjo's group in Japan by a subtractive hybridization technique in their pursuit of 
a novel protein involved in programmed cell death. ${ }^{7,8}$ The group used a murine 2B4.11 and LyD9 cell line which had been shown to die by a programmed cell death. PD-1 gene was isolated with subtractive hybridization using cDNA derived from 2B4.11 and LyD9.

As shown in Figure 1, PD-1 is a type I transmembrane glycoprotein with an estimated molecular weight of 50-55 kDa. Extracellular domain contains an immunoglobulin-like structure showing sequence homologies with those of other immune checkpoint inhibitors, like cytotoxic T-lymphocyte-associated protein 4 (CTLA4). Unlike other checkpoint inhibitors like CTLA4, PD-1 cannot homodimerize, since the membrane proximal cysteine residue does not exist in PD-1.

The PD-1 cytoplasmic domain is thought to transduce the negative signals into the cells through its immunoreceptor tyrosine-based inhibitory motif(ITIM) and immunoreceptor tyrosine-based switch motif (ITSM). ITIM and ITSM can bind with Src homology 2 domain-containing phosphatases, Src homology region 2 domain-containing phosphatase (SHP)-1, and SHP-2.

To evaluate the function of tyrosines in ITIM and ITSM of PD-1, Okazaki et al made constructs containing amino acid replacements in tyrosine residues in ITIM and/or ITSM. ${ }^{9}$ They transfected B-lymphocyte cell lines with each expression vector with PD-1 mutants and found that a tyrosine residue in ITSM but not in ITIM is necessary for the inhibitory signaling cascade from intracellular PD-1. The amino acid sequences around the ITSM of PD-1 were identical between human and mouse, suggesting that structures around the tyrosine in ITSM are important in negative regulation by PD-1. Phosphorylation of tyrosine in ITSM induces the binding of SHP-2 to PD-1, which in turn dephosphorylates and down-modulates the function of ZAP70 in T-lymphocytes (Figure 1). ${ }^{9}$

The PD-1 is expressed in a quite limited series of cells. PD-1 expression is observed in activated T-lymphocytes and T-cells with chronic stimulations. PD-1 expression is not found in naïve T-lymphocytes. Upon stimulation through $\mathrm{T}$-cell receptor and costimulation, nuclear factor of activated T-cells, cytoplasmic 1 (NFATc1) binds with DNA and induces the expression of PD-1 mRNA. IFN- $\alpha$ stimulation exerts IFN regulatory factor 9 binding with the promoter sequence of PD-1 gene and induces the PD-1 mRNA transcription.

In contrast to the limited expression of PD-1, PD-1 ligands - PD-L1 and PD-L2 - are widely expressed. PD-L1 and PD-L2 are type I transmembrane glycoproteins and belong to the B7 family. Other B7 family protein, CD80 and CD86, bind with CD28 and CTLA-4 and share sequence homology with PD-1. Extracellular domains of PD-L1 and PD-L2 have immunoglobulin-like structures. PD-L1 and PD-L2 have $40 \%$ amino acid homology between them. The functions of cytoplasmic regions of PD-L1 and PD-L2 are not well known.

PD-L1 is expressed in a wide variety of immune cells including activated T-lymphocytes, B-lymphocytes, dendritic cells, and macrophages. PD-L1 is also expressed in non-lymphoid cells or tissues, such as endothelial cells, lung cancer cells, and breast cancer cells.

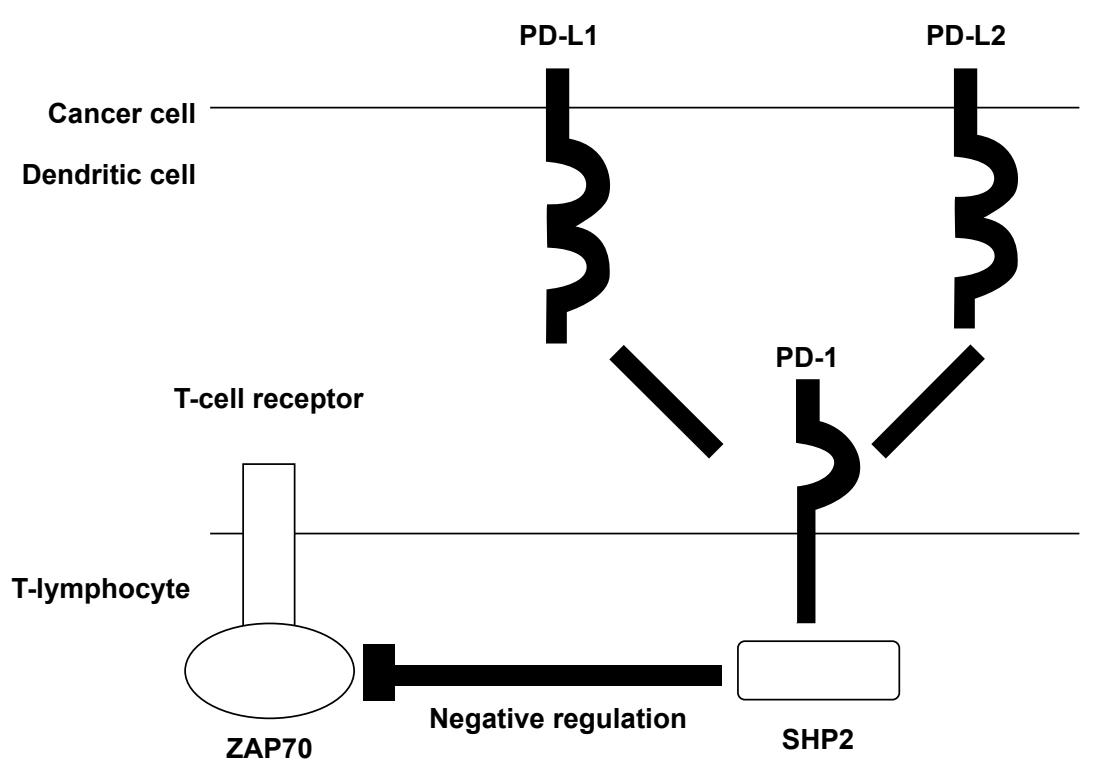

Figure I Molecular structures of PD-I and PD-I ligands, PD-LI and PD-L2.

Abbreviations: PD, programmed death receptor; SHP, Src homology region 2 domain-containing phosphatase. 
The PD-L2 protein is usually expressed in lymphoid tissues; however, its expression is also observed in some cancer cell lines and tissues as discussed later.

\section{Autoimmunity in PD-I-deficient mice}

To delineate biological roles of PD-1 in vivo, Nishimura et al generated PD-1 deficient mice by a gene-targeting strategy. ${ }^{10-12}$ On the C57BL/6 background, the serum level of IgG3 in PD-1-knockout mice was markedly elevated compared with that in control wild-type mice. Aged PD-1 deficient mice developed lupus-like glomerulonephritis. Histological examination of PD-1-deficient mice revealed the deposition of $\mathrm{IgG} 3$ and $\mathrm{C} 3$ in glomeruli.

Aged PD-1-deficient mice also developed arthritis with the proliferation of synovial cells histologically resembling rheumatoid arthritis.

When lpr mutation of the Fas gene was introduced into PD-1 deficient mice, they exhibited much more severe glomerulonephritis and arthritis. Moreover, massive lymphadenopathy took place in B6-lpr/lpr $\times$ PD-1 $1^{-/-}$mice.

When PD-1-deficient mice were backcrossed on BALB/c background, PD-1-deficient mice began to die as early as 5 weeks of age. Two-thirds of PD-1-deficient mice died by 30 weeks of age. Histological examination of the autopsy specimens of PD-1-deficient mice demonstrated that both ventricles were dilated and the right ventricle wall was thin. Functional assessment revealed that the movement of walls of the left ventricles was significantly decreased. IgG was deposited on the cardiomyocytes. Thus, PD-1-deficient mice suffered from autoimmune dilated cardiomyopathy with severely impaired contraction.

These results on the PD- 1 deficient mice with the several backgrounds indicated that loss of PD-1 led to the systemic autoimmunity in several organs. Moreover, also in humans, as discussed later in this paper, PD-1 blockade by anti-PD-1 antibody administrations leads to autoimmune adverse events, such as thyroiditis. Therefore, PD-1 is considered to play an important role in patrolling and preventing excessive immune reactions by negatively regulating the functions of autoreactive T-lymphocytes in a physiological state.

\section{Expression of PD-LI and PD-L2 in malignant tumors and PD-I in tumor-infiltrating lymphocytes}

As we mentioned above, in contrast to the limited expression of PD-1, PD-L1 is expressed in a wide variety of immune cells including activated T-lymphocytes, B-lymphocytes, dendritic cells, and macrophages. PD-L1 is also expressed in non-lymphoid cells or tissues, such as endothelial cells. To explore the expression level and pattern of PD-L1 in cancers, there have been several reports on the expression of PD-L1 in various types of malignant tumors using immunohistochemistry and other techniques.

There was a correlation between the expression of PD-L1 in renal cell carcinoma and stages and prognosis. ${ }^{13}$

The expression of PD-L1 in esophageal cancer and its association with prognosis was demonstrated by a Japanese group. ${ }^{14} \mathrm{PD}-\mathrm{L} 2$-positive patients also had a poorer prognosis than the negative patients.

Also, in gastric cancer, ovarian cancer, urothelial cancer, and pancreatic cancer, there is a correlation between the expression of PD-L1 and tumor stages and prognosis. ${ }^{15-18}$

Correlation between the expression of PD-L1 in malignant melanoma and tumor stages and prognosis was first studied by the group from Japan. ${ }^{19}$ Analysis of 59 clinical specimens of malignant melanoma revealed that patients with high PD-L1 immunoreactivity in tumors were at more advanced stage of malignant melanoma and were more likely to die from malignant melanoma than patients with low PD-L1 immunoreactivity. Another study by an Italian group also concluded that high PD-L1 expression in malignant melanoma indicates a poor prognosis. ${ }^{20}$

Taken together, the high expression of PD-L1 in tumors can be one of the prognostic factors in various malignant tumors indicating a worse survival rate. Moreover, in some tumors like esophageal cancer, high expression of PD-L2 in tumors is also correlated with poor prognosis.

The presence of PD-1-positive tumor-infiltrating lymphocytes is also associated with poor prognosis in various cancers, such as renal cancer and breast cancer. ${ }^{21,22}$

\section{Anti-PD-I antibody therapy in mice}

To explore the possibility of modulation of immune checkpoint inhibitor PD-1/PD-L1 system for tumor treatment, a Japanese group injected the mouse tumor cell lines into mice and compared the frequencies of tumor formation between wild-type mice and PD-1-deficient mice. ${ }^{23-24}$ P815 is a murine mastocytoma cell line which does not express PD-L1 or PD-L2. P815 was transfected with an expression PD-L1 vector. Forced expression of PD-L1 in P815 cells made the cells less susceptible to the cytotoxic activity of cytotoxic T-lymphocytes. Moreover, P815 cells with high PD-L1 tended to form tumors when injected into mice.

Another cell line, myeloma J558L cell line, was injected into wild-type or PD-1-deficient mice in a subsequent study. In J558L-injected wild-type mice, tumors grew rapidly, while tumor 
formation was suppressed in PD-1-deficient mice. The same group also injected B16 melanoma cells into the murine spleen in wild-type or PD-1 transgenic mice and found that tumors grew more rapidly in PD-1 transgenic mice than in wild-type mice. ${ }^{24}$

These results on PD-1 and PD-L1 in tumor immunity led to a development of a new therapy for human malignant tumors (Figure 2).

\section{Development of a PD-I-abrogating IgG4 monoclonal antibody: nivolumab}

Transgenic mice expressing human immunoglobulin loci were injected with PD-1-expressing cells and fully human anti-PD-1 antibodies were produced. ${ }^{25}$ Binding of each antibody with PD-1 and lack of binding with other immune checkpoint inhibitor was checked. A monoclonal anti-PD-1 antibody was chosen for its high affinity for PD-1. This fully human IgG4 anti-PD-1 antibody was changed into an IgG4 antibody with a hinge mutation (S228P) to reduce binding to Fc receptors (nivolumab).

\section{Nivolumab activates human melanoma antigen-specific cytotoxic T-lymphocytes}

Wong et al added the nivolumab into the culture medium and examined the number of cytotoxic T-lymphocytes against human melanoma associated antigens, melanoma antigen recognized by T-cells (MART)-1, and glycoprotein 100 (gp100). ${ }^{6,25-27}$ They found that administration of nivolumab resulted in the increase in the number of MART-1- or gp100responsive cytotoxic T-lymphocytes. Cytokine production by T-lymphocytes was also increased by the addition of nivolumab.

\section{Initial studies on nivolumab for malignant tumors}

Brahmer et al studied the effect of nivolumab for 39 patients with malignant tumors including colorectal cancer (CRC), advanced metastatic melanoma, prostate cancer, non-smallcell lung cancer, and renal cell carcinoma. ${ }^{28}$ Nivolumab was well tolerable and the serum half-life of nivolumab was found to be 12-20 days.

One patient with CRC showed durable complete response, and one patient with malignant melanoma and another patient with renal cell carcinoma showed partial responses.

Based upon the Phase I study on effects, safety, and tolerability of nivolumab against malignant tumors described above, Topalian et al studied the effects of nivolumab for 296 patients with malignant tumors. ${ }^{29}$

In total, $41 \%$ of the patients showed treatment-related adverse events: nine patients with pneumonitis, four patients with allergic rhinitis, 33 patients with diarrhea, 36 patients with
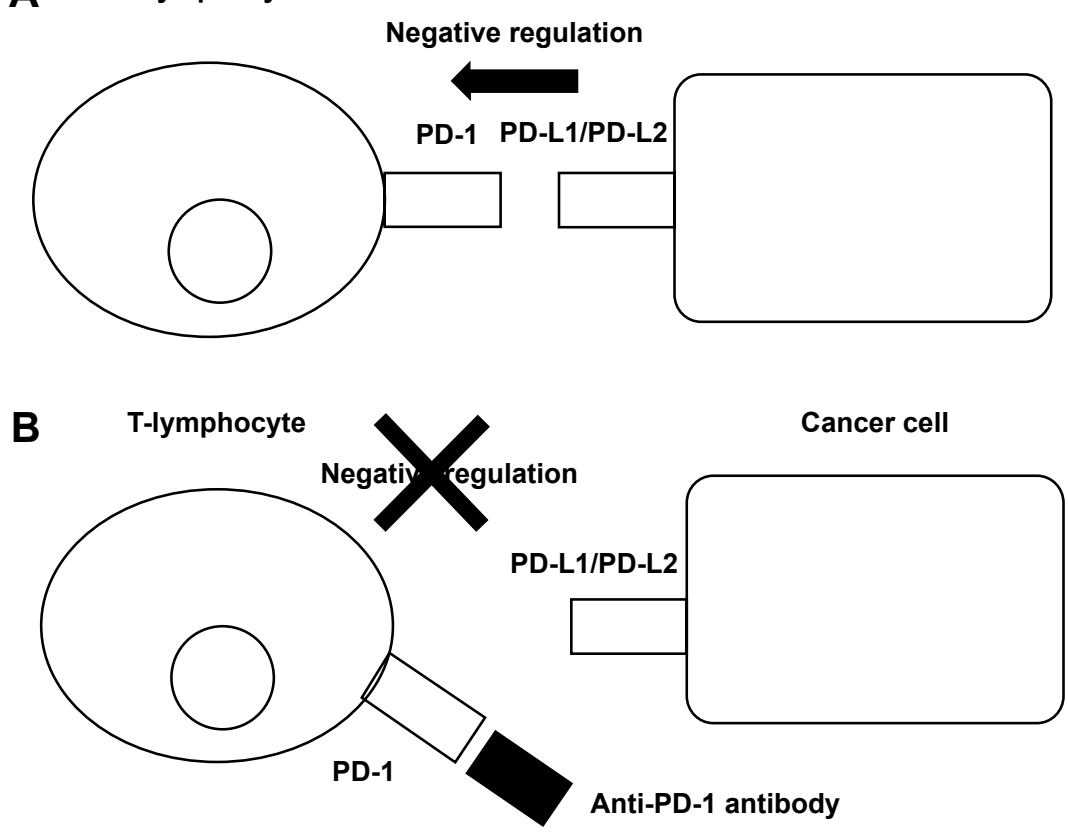

Figure 2 The mechanism of the effect of anti-PD-I antibody, nivolumab, for malignant tumors.

Note: Administration of an anti-PD-I antibody, nivolumab, blocks the negative regulation of tumor immunity by PD-I and PD-I ligands (PD-LI/PD-L2) (A and B). Abbreviation: PD, programmed death receptor. 
rash, 28 patients with pruritus, eleven patients with increased alanine aminotransferase, nine patients with increased thyroid-stimulating hormone, eight patients with aspartate aminotransferase, seven patients with hypothyroidism, three patients with hyperthyroidism, and nine cases with infusionrelated reaction or hypersensitivity.

Objective responses (complete response or partial response) were observed in 26 of 94 patients $(29 \%, 19 \%$, $30 \%, 41 \%$, and $20 \%$ at a dose of $0.1 \mathrm{mg} / \mathrm{kg}, 0.3 \mathrm{mg} / \mathrm{kg}$, $1.0 \mathrm{mg} / \mathrm{kg}, 3.0 \mathrm{mg} / \mathrm{kg}$, and $10.0 \mathrm{mg} / \mathrm{kg}$, respectively). Progression-free survival rate at 24 weeks was $41 \%$ for malignant melanoma patients.

The median PD-1 receptor occupancy rate in peripheral blood mononuclear cells by nivolumab after one cycle of nivolumab treatment was $64 \%-70 \%$ for malignant melanoma patients.

Immunohistochemical study revealed that 25 of the 42 examined malignant tumor specimens were positive for PD-L1 expression. Of these 25 patients, nine (36\%) had an objective response, whereas there was no objective response among the 17 patients with PD-L1-negative tumors, indicating that nivolumab is more likely to be effective for PD-L1positive tumors than for PD-L1-negative tumors.

Several things have been kept in mind when interpreting these data. First, the expression of PD-L1 can be increased by phosphoinositide- 3 kinase and other pathways irrelevant of T-cell infiltration or antitumor immune response. In this case, PD-L1-positive tumors will not be necessarily antiPD1 therapy.

Second, some patients without PD-L1 expression have been shown to respond to anti-PD1 therapy in other reports. There are several possible explanations for this phenomenon. First, there is a heterogeneity of the tumor in the expression level of PD-L1 and tumor biopsies are not representative of the whole tumor. Secondly, the expression of PD-L1 is dynamic and inducible by some cytokines like IFN- $\gamma$ secreted by immune cells. Therefore, reactivation of $\mathrm{CD}^{+} \mathrm{T}$-cells in the tumor will induce higher expression of PD-L1 compared to the baseline expression of PD-L1, when tumor biopsies are performed. Thirdly, due to the technical limitation of immunohistochemical PD-L1 detection system, some weakly PD-L1-expressing tumors can be classified into PD-L1-negative tumors.

\section{Combination therapy with nivolumab and ipilimumab for melanoma}

Ipilimumab is a fully human IgG1 monoclonal antibody against another immune checkpoint inhibitor, CTLA4. Administration of ipilimumab for advanced melanoma improved overall survival. ${ }^{30,31}$ Wolchok et al conducted a Phase I trial of nivolumab combined with ipilimumab for advanced melanoma. ${ }^{32}$

In total, 86 patients were enrolled in the study. Fifty-three patients received nivolumab and ipilimumab every 3 weeks for 12 weeks and nivolumab alone (concurrent regimen). Thirty-three patients pretreated with ipilimumab received nivolumab every 2 weeks (sequenced regimen).

Among the concurrent regimen group, 21 (40\%) out of 53 patients showed objective responses. Among the sequenced regimen group, six $(20 \%)$ out of 30 patients showed objective responses.

Based upon the above-mentioned result of the Phase I study of combination therapy of nivolumab and ipilimumab, Postow et al performed a double-blind study and concluded that combination therapy with nivolumab and ipilimumab is statistically significantly more effective than an ipilimumab monotherapy. ${ }^{33}$

\section{Combination therapy with nivolumab and vaccine for malignant melanoma}

Weber et al examined the effect of nivolumab with or without a peptide vaccine for malignant melanoma. ${ }^{34}$ Ninety patients with stage III or IV advanced malignant melanoma were enrolled in this study. Objective response rate for nivolumab with or without vaccine was $25 \%$. Objective response rate in PD-L1 tumor-positive group was $67 \%$ and that in PD-L1 tumor-negative group was $19 \%$. The effect of combination with vaccine was not fully elucidated in this study.

\section{Durable effect of nivolumab for malignant melanoma}

Topalian et al studied the durable effect of nivolumab for malignant melanoma. ${ }^{35}$ One hundred and seven patients with malignant melanoma were enrolled in this study. Median overall survival was 16.8 months. Objective response rate was $31 \%$.

In total, there were adverse events in 90 (84.1\%) out of 107 patients: fatigue, pyrexia, pain, rash, pruritus, vitiligo, acneiform dermatitis, photosensitivity reaction, diarrhea, nausea, abdominal pain, dry mouth, vomiting, decreased appetite, hypothyroidism, and infusion-related reaction.

\section{Comparison of the effect of nivolumab for malignant melanoma with other chemotherapies}

Recent studies compared the effects and adverse events of nivolumab and other chemotherapies. ${ }^{36,37}$ 
The effect of nivolumab was compared with that of dacarbazine for malignant melanoma patients without $B R A F$ mutation. ${ }^{36}$ Objective response rate for nivolumab-treated patients was $40.0 \%$, while that for dacarbazine-treated patients was $13.9 \%$. The rate of any adverse events in nivolumabtreated patients was $74.3 \%$, whereas that for dacarbazinetreated patients was $75.6 \%$.

Weber et al compared nivolumab with other chemotherapies, dacarbazine or paclitaxel with carboplatin, in patients unresponsive to anti-CTLA-4 treatment. ${ }^{37}$ Objective response rate for nivolumab was $31 \%$ and that for dacarbazine or paclitaxel with carboplatin was $10.7 \%$.

Therefore, nivolumab has been well demonstrated to show better response for malignant melanoma than the existent chemotherapy, dacarbazine.

\section{Conclusion}

There are a lot of patients with advanced melanoma who are resistant to conventional chemotherapies with an alkylating agent, dacarbazine, and IFN- $\alpha$. Recent studies have revealed that nivolumab with or without ipilimumab or vaccines can become an alternative therapy for those patients. Moreover, recent papers showed nivolumab treatment showed superior prognostic outcome than that of dacarbazine for advanced malignant melanoma. Based upon the findings mentioned above, nivolumab may be a well tolerable and durable therapy for an advanced melanoma.

Pembrolizumab is another humanized anti-PD1 antibody and blocks the interaction between PD1 and its ligands. Pembrolizumab has also been approved for treating advanced melanoma in USA. ${ }^{38}$

Expression of PD-L1 in tumors can be a good indicator for objective responses to nivolumab; however, further analysis is required to find other biomarkers in the serum to anticipate the outcomes of nivolumab in future.

\section{Disclosure}

The authors report no conflicts of interest in this work.

\section{References}

1. Deeks ED. Nivolumab: a review of its use in patients with malignant melanoma. Drugs. 2014;74(11):1233-1239.

2. Okura R, Yoshioka H, Yoshioka M, Hiromasa K, Nishio D, Nakamura M. Expression of AID in malignant melanoma with BRAF(V600E) mutation. Exp Dermatol. 2014;23(5):347-348.

3. Topalian SL, Weiner GJ, Pardoll DM. Cancer immunotherapy comes of age. J Clin Oncol. 2011;29(36):4828-4836.

4. Mellman I, Coukos G, Dranoff G. Cancer immunotherapy comes of age. Nature. 2011;480(7378):480-489.

5. Okazaki T, Chikuma S, Iwai Y, Fagarasan S, Honjo T. A rheostat for immune responses: the unique properties of PD-1 and their advantages for clinical application. Nat Immunol. 2013;14(12):1212-1218.
6. Lu J, Lee-Gabel L, Nadeau MC, Ferencz TM, Soefje SA. Clinical evaluation of compounds targeting PD-1/PD-L1 pathway for cancer immunotherapy. J Oncol Pharm Pract. Epub 2014 Jun 9.

7. Ishida Y, Agata Y, Shibahara K, Honjo T. Induced expression of PD-1, a novel member of the immunoglobulin gene superfamily, upon programmed cell death. EMBO J. 1992;11(11):3887-3895.

8. Okazaki T, Honjo T. PD-1 and PD-1 ligands: from discovery to clinical application. Int Immunol. 2007;19(7):813-824.

9. Okazaki T, Maeda A, Nishimura H, Kurosaki T, Honjo T. PD-1 immunoreceptor inhibits $\mathrm{B}$ cell receptor-mediated signaling by recruiting src homology 2-domain-containing tyrosine phosphatase 2 to phosphotyrosine. Proc Natl Acad Sci U S A. 2001;98(24):13866-13871.

10. Nishimura H, Minato N, Nakano T, Honjo T. Immunological studies on PD-1 deficient mice: implication of PD-1 as a negative regulator for B cell responses. Int Immunol. 1998;10(10):1563-1572.

11. Nishimura H, Nose M, Hiai H, Minato N, Honjo T. Development of lupus-like autoimmune diseases by disruption of the $P D-1$ gene encoding an ITIM motif-carrying immunoreceptor. Immunity. 1999;11(2): 141-151.

12. Nishimura H, Okazaki T, Tanaka Y, et al. Autoimmune dilated cardiomyopathy in PD-1 receptor-deficient mice. Science. 2001;291(5502): 319-322.

13. Thompson RH, Gillett MD, Cheville JC, et al. Costimulatory B7-H1 in renal cell carcinoma patients: Indicator of tumor aggressiveness and potential therapeutic target. Proc Natl Acad Sci U S A. 2004;101(49): 17174-17179.

14. Ohigashi Y, Sho M, Yamada Y, et al. Clinical significance of programmed death-1 ligand-1 and programmed death-1 ligand-2 expression in human esophageal cancer. Clin Cancer Res. 2005;11(8):2947-2953.

15. Wu C, Zhu Y, Jiang J, Zhao J, Zhang XG, Xu N. Immunohistochemical localization of programmed death-1 ligand-1 (PD-L1) in gastric carcinoma and its clinical significance. Acta Histochem. 2006;108(1): $19-24$.

16. Hamanishi J, Mandai M, Iwasaki M, et al. Programmed cell death 1 ligand 1 and tumor-infiltrating CD8+ T lymphocytes are prognostic factors of human ovarian cancer. Proc Natl Acad Sci U S A. 2007;104(9): $3360-3365$.

17. Nakanishi J, Wada Y, Matsumoto K, Azuma M, Kikuchi K, Ueda S. Overexpression of B7-H1 (PD-L1) significantly associates with tumor grade and postoperative prognosis in human urothelial cancers. Cancer Immunol Immunother. 2007;56(8):1173-1182.

18. Nomi T, Sho M, Akahori T, et al. Clinical significance and therapeutic potential of the programmed death-1 ligand/programmed death-1 pathway in human pancreatic cancer. Clin Cancer Res. 2007;13(7):2151-2157.

19. Hino R, Kabashima K, Kato Y, et al. Tumor cell expression of programmed cell death-1 ligand 1 is a prognostic factor for malignant melanoma. Cancer. 2010;116(7):1757-1766.

20. Massi D, Brusa D, Merelli B, et al. PD-L1 marks a subset of melanomas with a shorter overall survival and distinct genetic and morphological characteristics. Ann Oncol. 2014;25(12):2433-2442.

21. Thompson RH, Dong H, Lohse CM, et al. PD-1 is expressed by tumorinfiltrating immune cells and is associated with poor outcome for patients with renal cell carcinoma. Clin Cancer Res. 2007;13(6):1757-1761.

22. Muenst S, Soysal SD, Gao F, Obermann EC, Oertli D, Gillanders WE. The presence of programmed death 1 (PD-1)-positive tumor-infiltrating lymphocytes is associated with poor prognosis in human breast cancer. Breast Cancer Res Treat. 2013;139(3):667-676.

23. Iwai Y, Terawaki S, Honjo T. PD-1 blockade inhibits hematogenous spread of poorly immunogenic tumor cells by enhanced recruitment of effector T cells. Int Immunol. 2005;17(2):133-144.

24. Iwai Y, Ishida M, Tanaka Y, Okazaki T, Honjo T, Minato N. Involvement of PD-L1 on tumor cells in the escape from host immune system and tumor immunotherapy by PD-L1 blockade. Proc Natl Acad Sci US A. 2002;99(19):12293-12297.

25. Wong RM, Scotland RR, Lau RL, et al. Programmed death-1 blockade enhances expansion and functional capacity of human melanoma antigen-specific CTLs. Int Immunol. 2007;19(10):1223-1234. 
26. O’Sullivan Coyne G, Madan RA, Gulley JL. Nivolumab: promising survival signal coupled with limited toxicity raises expectations. J Clin Oncol. 2014;32(10):986-988.

27. McDermott DF, Atkins MB. PD-1 as a potential target in cancer therapy. Cancer Med. 2013;2(5):662-673.

28. Brahmer JR, Drake CG, Wollner I, et al. Phase I study of single-agent anti-programmed death-1 (MDX-1106) in refractory solid tumors: safety, clinical activity, pharmacodynamics, and immunologic correlates. J Clin Oncol. 2010;28(19):3167-3175.

29. Topalian SL, Hodi FS, Brahmer JR, et al. Safety, activity, and immune correlates of anti-PD-1 antibody in cancer. NEngl J Med. 2012;366(26): 2443-2454.

30. Hodi FS, O'Day SJ, McDermott DF, et al. Improved survival with ipilimumab in patients with metastatic melanoma. $N$ Engl J Med. 2010;363(8):711-723.

31. Robert C, Thomas L, Bondarenko I, et al. Ipilimumab plus dacarbazine for previously untreated metastatic melanoma. $N$ Engl J Med. 2011;364(26):2517-2526.

32. Wolchok JD, Kluger H, Callahan MK, et al. Nivolumab plus ipilimumab in advanced melanoma. $N$ Engl J Med. 2013;369(2):122-133.
33. Postow MA, Chesney J, Pavlick AC, et al. Nivolumab and ipilimumab versus ipilimumab in untreated melanoma. NEngl J Med. 2015;372(21): 2006-2017.

34. Weber JS, Kudchadkar RR, Yu B, et al. Safety, efficacy, and biomarkers of nivolumab with vaccine in ipilimumab-refractory or -naive melanoma. J Clin Oncol. 2013;31(34):4311-4318.

35. Topalian SL, Sznol M, McDermott DF, et al. Survival, durable tumor remission, and long-term safety in patients with advanced melanoma receiving nivolumab. J Clin Oncol. 2014;32(10):1020-1030.

36. Robert C, Long GV, Brady B, et al. Nivolumab in previously untreated melanoma without BRAF mutation. $N$ Engl J Med. 2015;372(4): 320-330.

37. Weber JS, D'Angelo SP, Minor D, et al. Nivolumab versus chemotherapy in patients with advanced melanoma who progressed after anti-CTLA-4 treatment (CheckMate 037): a randomized, controlled, open-label, phase 3 trial. Lancet Oncol. 2015;16(4):375-384.

38. Gangadhar TC, Salama AKS. Clinical applications of PD-1-based therapy: a focus on pembrolizumab (MK-3475) in the management of melanoma and other tumor types. Onco Targets Ther. 2015;8:929-937.

\section{Publish your work in this journal}

OncoTargets and Therapy is an international, peer-reviewed, open access journal focusing on the pathological basis of all cancers, potential targets for therapy and treatment protocols employed to improve the management of cancer patients. The journal also focuses on the impact of management programs and new therapeutic agents and protocols on

\section{Dovepress}

patient perspectives such as quality of life, adherence and satisfaction. The manuscript management system is completely online and includes a very quick and fair peer-review system, which is all easy to use. Visit http://www.dovepress.com/testimonials.php to read real quotes from published authors. 